\title{
MRI-guided coupling for a focused ultrasound system using a top-to-bottom propagation
}

\author{
Marinos Yiannakou', George Menikou², Christos Yiallouras ${ }^{1,3}$ and Christakis Damianou ${ }^{1 *}$ (D)
}

\begin{abstract}
Background: A novel magnetic resonance imaging (MRI)-conditional coupling system was developed that accommodates a focused ultrasound (FUS) transducer. With this coupling system, the transducer can access targets from top to bottom. The intended clinical application is treatment of fibroids using FUS with the patient placed in supine position.

Methods: The coupling system was manufactured using a rapid prototyping device using acrylonitrile butadiene styrene (ABS) plastic. Coupling to a gel phantom was achieved using a water bag filled with degassed water. The FUS transducer was immersed in the water bag.

Results: The coupling system was successfully tested for MRI compatibility using fast-gradient pulse sequences in a gel phantom. The robotic system with its new coupling system was evaluated for its functionality for creating discrete and multiple (overlapping) lesions in the gel phantom.

Conclusions: An MRI-conditional FUS coupling system integrated with an existing robotic system was developed that has the potential to create thermal lesions in targets using a top-to-bottom approach. This system has the potential to treat fibroid tumors with the patient lying in supine position.
\end{abstract}

Keywords: Ultrasound, Robot, MRI, Fibroid, Cancer

\section{Background}

The available main treatment options for uterine fibroids include hysterectomy, myomectomy, and uterine artery embolization. Hysterectomy is the primary option for resolving fibroid-associated symptoms. Uterine artery embolization (UAE) is major treatment option for fibroids which was introduced in 1995 [1]. This treatment option involves femoral artery catheterization and intra-arterial infusion of embolization particles. As a result, UAE produces ischemia of the fibroid uterus, thus reducing significantly the volume of fibroids [1]. Another treatment option for uterine fibroids involves hormonal manipulation. Gonadotrophin-releasing hormone $(\mathrm{GnRH})$ is predominantly used for the temporary reduction of fibroid volume by as much as $60 \%$ sometimes [2].

\footnotetext{
* Correspondence: cdamianou@cytanet.com.cy

${ }^{1}$ Cyprus University of Technology, Limassol, Cyprus

Full list of author information is available at the end of the article
}

Another treatment option which is not widely accepted is cryoablation [3]. With cryoablation, the uterine fibroid is cooled to very low temperatures. This option is introduced using either laparoscopic or hysteroscopic access $[4,5]$.

Another option deployed recently is magnetic resonance imaging-guided focused ultrasound (MRgFUS) [6]. MRgFUS is an effective and completely noninvasive modality. MRgFUS may be used as a fertility-preserving option for some cases. The first treatment of uterine fibroids using MRgFUS was performed in 2003 and was implemented by Stewart and colleagues [6]. The results of this study were promising, and thus lead to additional clinical trials. The goal of the additional trials was to evaluate the efficacy of MRgFUS in larger number of patients. These studies showed significant reduction of clinical symptoms. Additionally, improvement in life quality was reported at 6,12 , and 24 months. Until now, more than 8500 patients have been treated with MRgFUS 
worldwide. Only few side effects were reported [7]. The acceptance rates from patients are evaluated as high [7]. In recent reports of clinical trials, with proper selection of patient population, the reduction in volume is often more than $50 \%$ [8]. However, despite the good clinical results, one of the disadvantages of this procedure is the lengthy procedure time (i.e., low time efficiency) which is a big problem when treating large fibroids.

The first MRgFUS commercial system (Exablate) to be used for the treatment of fibroids was developed by InSightec (Tirat Carmel, Israel) [9]. The system uses a phased-array transducer operating close to $1 \mathrm{MHz}$. The transducer is positioned close to the target using a robotic system. The whole system is placed in the magnetic resonance imaging (MRI) table. Treatment is performed with the patient in the prone position and under light sedation, with active monitoring of vital signs.

In 2009, Philips developed the MRgFUS robotic system (Sonalleve, Philips Healthcare) that was Conformité Européenne (CE)-marked for the treatment of uterine fibroids [10, 11]. Treatments were performed using a phased-array 256-channel transducer (radius of curvature $12 \mathrm{~cm}$, aperture $13 \mathrm{~cm}$; operable at $1.2 \mathrm{MHz}$ ) equipped with a mechanical displacement device with 5 degrees of freedom (three linear and two angular). This system is able to perform volumetric ablation. With this system, the patient is also placed in prone position.

This study includes the conversion of a robotic system intended for brain applications to a robotic system that can be used for accessing fibroids. This is achieved by modifying the coupling system, thus allowing top-tobottom coupling (axial in MRI). The system was evaluated in a gel phantom for producing discrete and overlapping lesions. The system uses a single-element transducer, which makes the system less complex and cost-effective compared to systems that use phasedarray technology.

\section{Methods}

\section{Coupling for fibroids}

An existing positioning device with three axes $(X, Y, Z)$ was used [12]. Figure 1 shows the existing robotic system dedicated for the brain. The coupling system was modified so that top-to-bottom access was possible. A modified arm was developed that was inserted in a coupling structure that makes coupling to fibroids (in this study, gel phantom). The three axes were driven by piezoelectric ultrasonic motors (USR60-S3N, Shinsei Kogyo Corp., Tokyo, Japan). Optical encoders were used (US Digital Corporation, Vancouver, WA 98684, USA). The encoder output was connected to the counter input of a data acquisition board USB 6251 (NI, Austin, USA). Figure 2 shows the developed coupling that can be used for a top-to-bottom access of ultrasound to targets. This

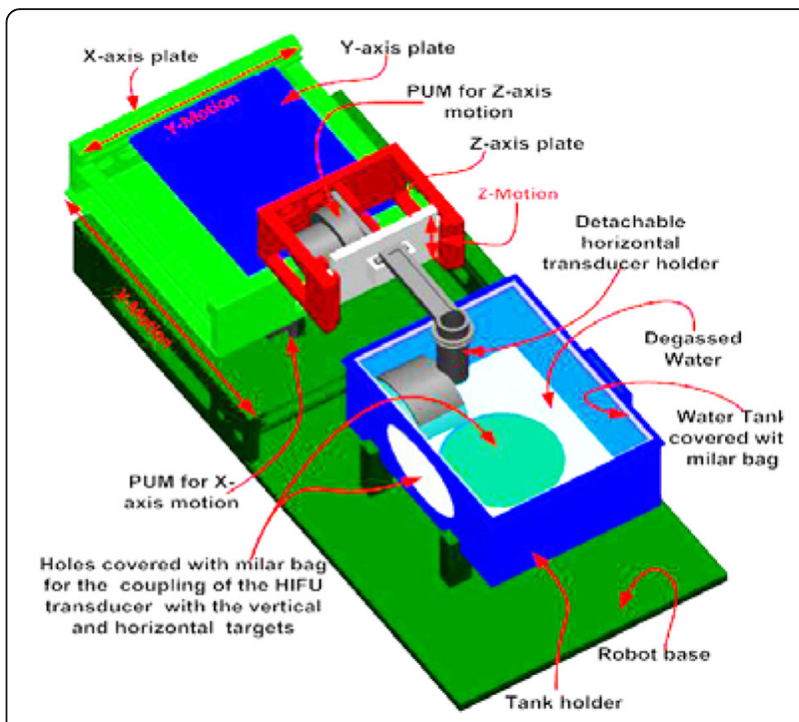

Fig. 1 The existing robotic system dedicated for the brain

prototype coupling system includes a transducer arm which is connected to the $Z$-axis, a holder for the water bag, and a base that holds the water bag holder. This coupling system is manually positioned to the patient. This structure includes a movable water bag (steps of $1 \mathrm{~cm}$ ), a transducer holder, and an arm that is fixed to the existing robot. Figure 3 shows the concept of using the modified robotic system for access to the fibroids.

\section{Experimental setup}

The arm of the robotic system with the 1-MHz transducer was immersed inside the water bag. The water bag was filled with degassed water. The transducer was placed above the gel phantom. The distance of the transducer from the phantom was such that the beam focus was placed in the middle of the gel phantom. The phantom was wrapped around by the GPFLEX coil (USA instruments, Cleveland, OH, USA) to perform all the imaging studies.

\section{FUS system}

The effectiveness of the system was evaluated by creating lesions in polyacrylamide gel phantom (ONDA Corporation, Sunnyvale, CA, USA). The FUS system consists of an RF amplifier (RFG 750W, JJA instruments, Seattle, WA, USA) and a spherical transducer made from piezoelectric ceramic (Sonic Concepts, USA). The transducer operates at $1.14 \mathrm{MHz}$ and has focal length of $10 \mathrm{~cm}$ and diameter of $3 \mathrm{~cm}$. The acoustical power of $20 \mathrm{~W}$ was applied in continuous mode for $60 \mathrm{~s}$. With $20 \mathrm{~W} / 60 \mathrm{~s}$, the goal was to get temperature maps and test the MR thermometry without damaging the gel permanently. In another exposure that creates lesions, the power used was $30 \mathrm{~W}$ for $30 \mathrm{~s}$. With this transducer, 


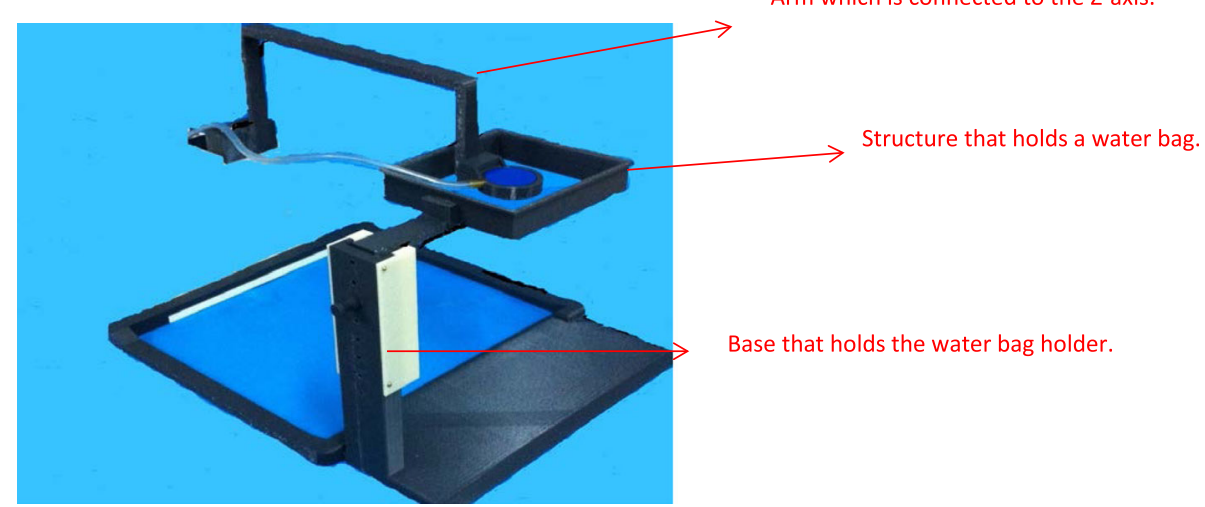

Fig. 2 The developed coupling that can be used for a top-to-bottom access of ultrasound to targets

and focal depth for $30 \mathrm{~W}$, lesions are created with a 20 -s exposure or higher. The heating of the system was evaluated in the gel phantom. Degassed water was placed between the transducer, water bag, and the gel phantom, thus providing good acoustical coupling between the gel phantom and the FUS transducer. The attenuation of the gel as reported by the manufacturer was $0.6 \mathrm{~dB} / \mathrm{cm}$ at $1 \mathrm{MHz}[13]$.

\section{MR imaging}

The robotic FUS system was tested in a 1.5-T MR system (Signa, General Electric, Fairfield, CT, USA) using a lumbar spine coil (USA instruments, Cleveland, $\mathrm{OH}$, USA).

\section{MR thermometry}

The temperature elevation during FUS exposures was estimated using the proton resonance frequency (PRFS) shift method [14]. This method relates the phase shift derived from the frequency shift of the MR signal due to the local temperature elevation $(\Delta T)$. This relationship is described by the following:

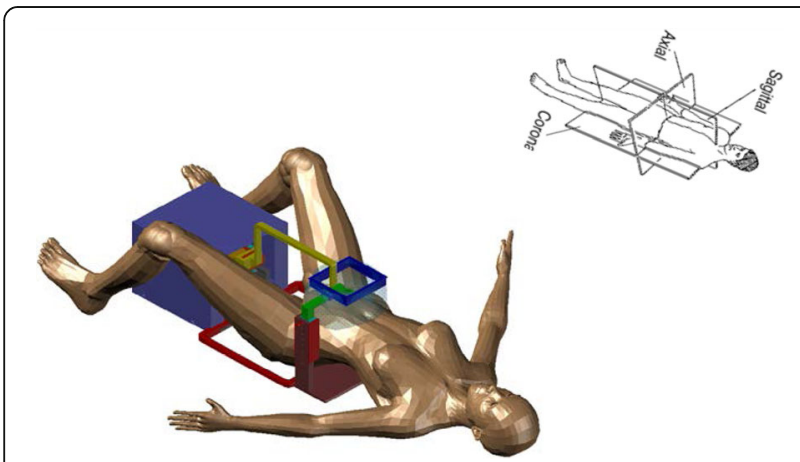

Fig. 3 The concept of using the modified robotic system for access to the fibroids

$$
\Delta T=\frac{\phi(T)-\phi\left(T_{0}\right)}{\gamma \alpha B_{0} \mathrm{TE}}
$$

where $\phi(T)$ and $\phi\left(T_{0}\right)$ are the absolute phases of the MR signal at a starting and final temperature $T$ and $T_{0}$, respectively; $\gamma$ is the gyromagnetic ratio; $\alpha$ is the PRF change coefficient $\left(0.01 \mathrm{ppm} /{ }^{\circ} \mathrm{C}\right) ; B_{0}$ is the magnetic field strength; and TE is the echo time.

The spoiled gradient echo sequence (SPGR) was used for thermometry: repetition time (TR) $38.5 \mathrm{~ms}$, TE $20 \mathrm{~ms}$, bandwidth $(\mathrm{BW}) 15 \mathrm{kHz}$, matrix $128 \times 128$, slice thickness: $10 \mathrm{~mm}$, and number of excitations (NEX): 1 . The temporal resolution of thermometry was about $12 \mathrm{~s}$. Phase maps were reconstructed by calculating the phase on a pixel-by-pixel basis after combining pixel data from real and imaginary channels. Although the scanner was capable of producing directly phase image reconstructions, the applied intra-scan gradient non-linearity corrections induce phase interpolation problems. All of the image processing was performed with custom-made software developed in MATLAB (MathWorks, Natick, USA). Temperature color-coded maps were produced by adjusting the color map (blue to red) for a range of minimum to maximum region of interest (ROI) temperature. Figure 4 shows the flowchart of the software that estimates temperature using the PRFS method.

High-resolution MR imaging was performed to visualize the FUS lesions in the gel phantom using T2-weighted fast spin echo (FSE) sequence (imaging parameters, TR $2500 \mathrm{~ms}$, TE $60 \mathrm{~ms}$, slice thickness $3 \mathrm{~mm}$, matrix $256 \times$ 256, field of view (FOV) $16 \mathrm{~cm}, \mathrm{NEX} 3$, and echo train length (ETL) 8).

\section{Results}

Figure 5 shows the MR image using T2-weighted (T2-W) FSE of the coupling to the gel of the robotic system. In this image, the transducer-water bag-gel phantom 


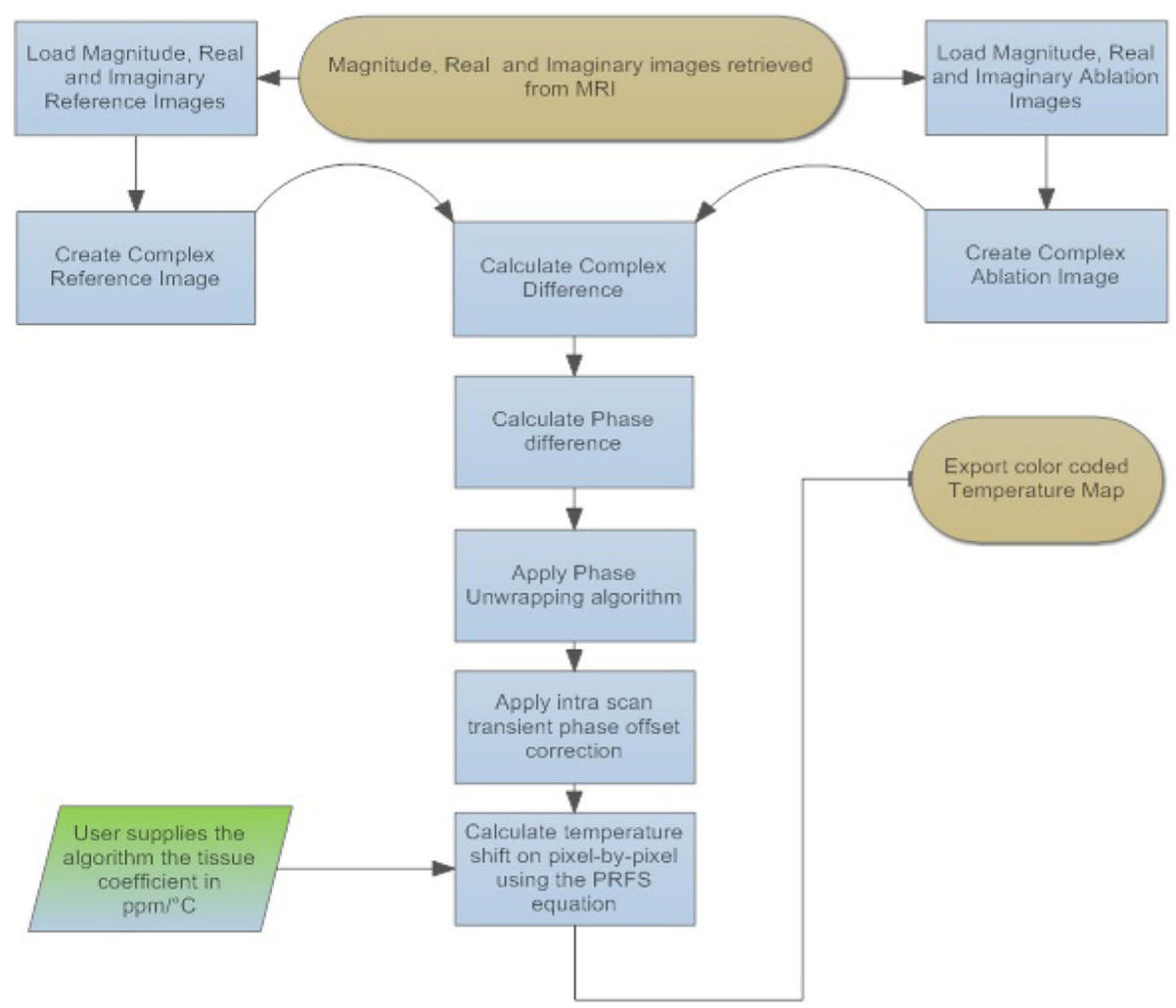

Fig. 4 Software flowchart for estimating MR thermometry

arrangement is shown demonstrating the excellent coupling to the gel phantom.

Figure 6 shows the axial temperature maps produced by this transducer. The acoustical power of $20 \mathrm{~W}$ was applied for $60 \mathrm{~s}$. During the first five images, the FUS transducer was activated. Having observed the focal beam in a plane perpendicular to the transducer face, the next step was to evaluate the temperature maps in a plane parallel to the transducer face. Figure 7 shows the coronal temperature maps produced by this transducer. The acoustical power of $20 \mathrm{~W}$ was applied for $60 \mathrm{~s}$. During the first five images, FUS was activated.

Figure 8 shows the MR images (using T2-W FSE) of three discrete thermal lesions created in the gel phantom by moving the $\mathrm{X}$ stage of the robotic system. The acoustical power used was $30 \mathrm{~W}$ for $30 \mathrm{~s}$. The spatial step between lesions was $5 \mathrm{~mm}$. With the transducer and focal depth with a $30 \mathrm{~W}$ and $30 \mathrm{~s}$ exposure, the lesion

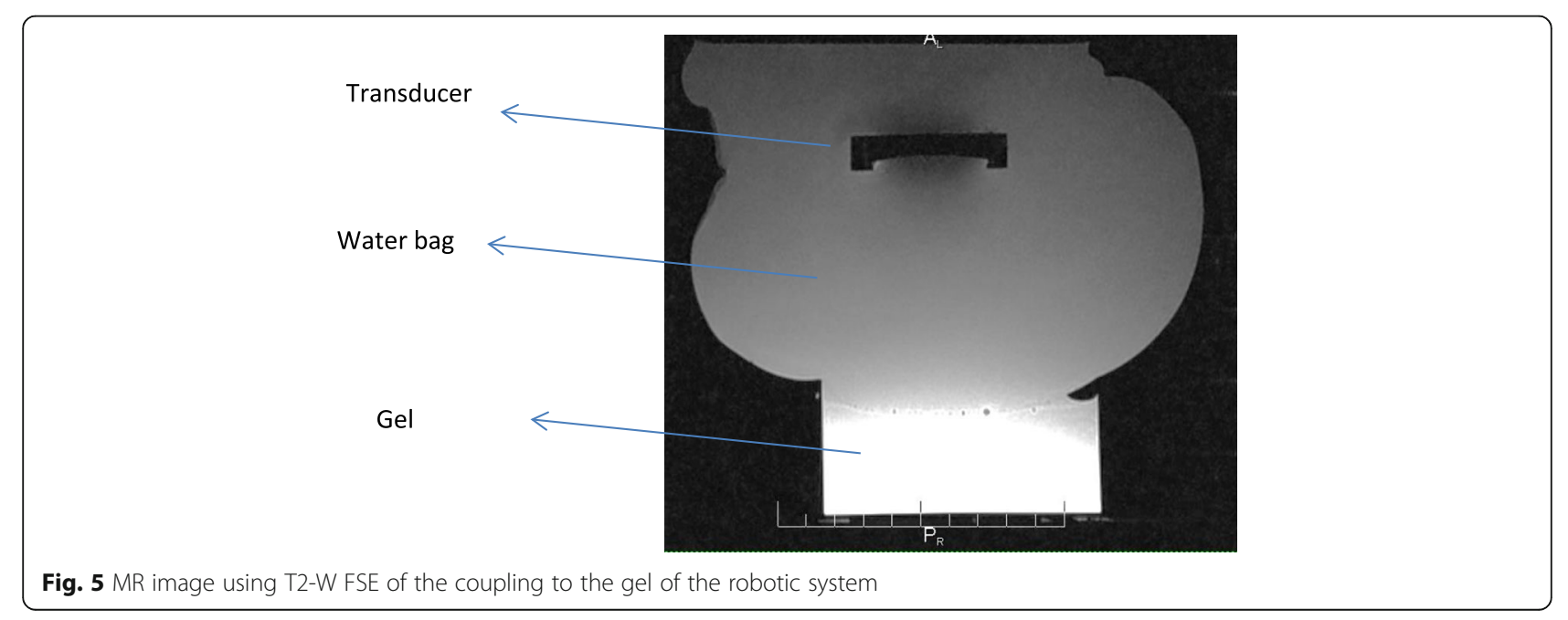



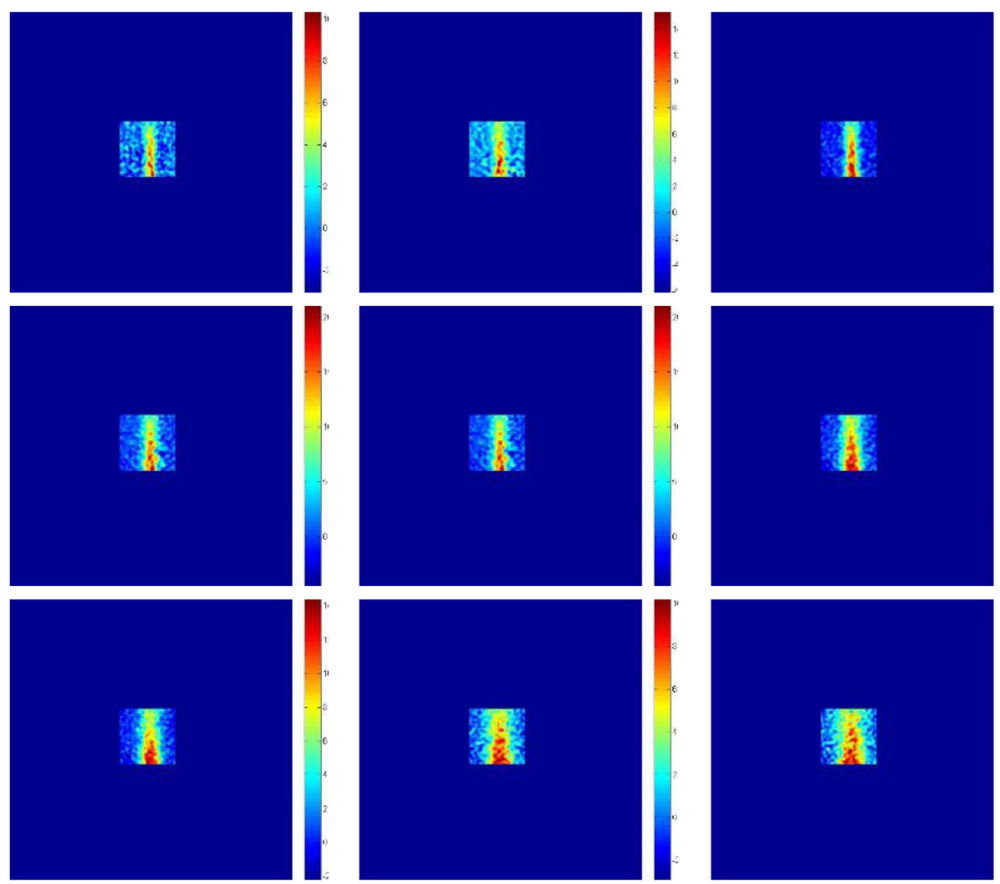

Fig. 6 Axial temperature maps produced by this transducer in a plane perpendicular to the face of the transducer. The acoustical power of $20 \mathrm{~W}$ was applied for $60 \mathrm{~s}$

width is $3.3 \mathrm{~mm}$, and the lesion length is $24 \mathrm{~mm}$ (maximum temperature recorded was $82^{\circ} \mathrm{C}$ ). With $60 \mathrm{~s}$ exposure and $30 \mathrm{~W}$, the lesion width is $4.2 \mathrm{~mm}$ and the lesion length is $28.4 \mathrm{~mm}$ (maximum temperature recorded was $94{ }^{\circ} \mathrm{C}$ ). At higher power, the lesion size does not change much since conduction carries the heat away. Also, a higher power will cause the temperature to exceed $100{ }^{\circ} \mathrm{C}$ (tissue boiling point). Figure 9 shows the MR image (using T2-W FSE) of the three discrete thermal lesions of Fig. 8 in axial plane demonstrating the penetration deep in the gel (plane perpendicular to the transducer face).
Figure 10 shows the MR images (using T2-W FSE) of four overlapping thermal lesions created in the gel phantom by moving the $\mathrm{X}$ and $\mathrm{Y}$ stages of the robotic system in a $2 \times 2$ square grid. The acoustical power used was $30 \mathrm{~W}$ for $30 \mathrm{~s}$. The spatial step between lesions was $3 \mathrm{~mm}$. Because the width of the lesion is close to $3 \mathrm{~mm}$ (see Fig. 8), then to get overlapping lesions, the step size had to be $3 \mathrm{~mm}$. This figure clearly demonstrates the effectiveness of the positioning device for creating large lesions for the purpose of thermal ablation.
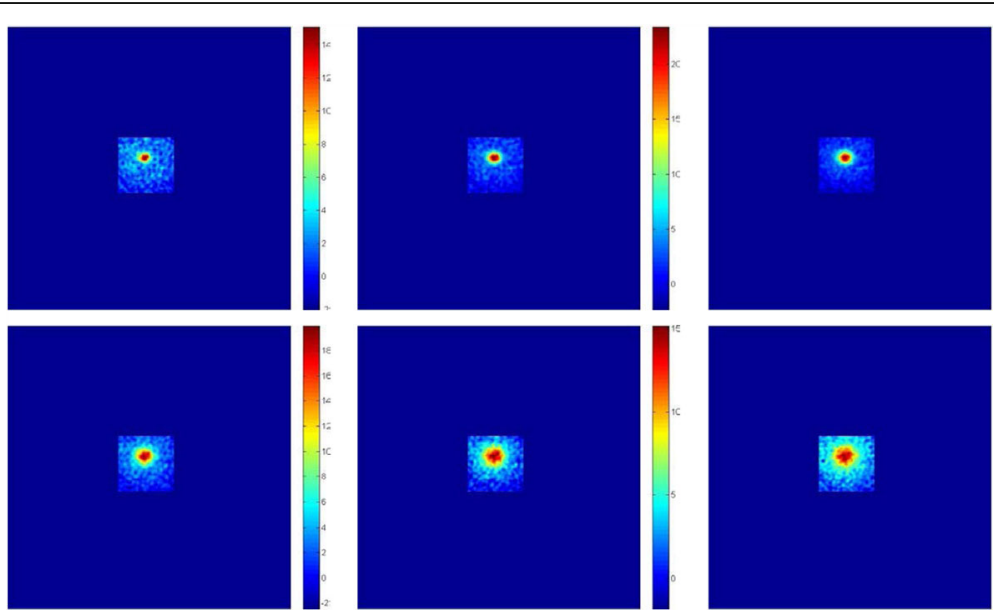

Fig. 7 Coronal temperature maps produced by this transducer in a plane parallel to the face of the transducer. The acoustical power of $20 \mathrm{~W}$ was applied for $60 \mathrm{~s}$ 

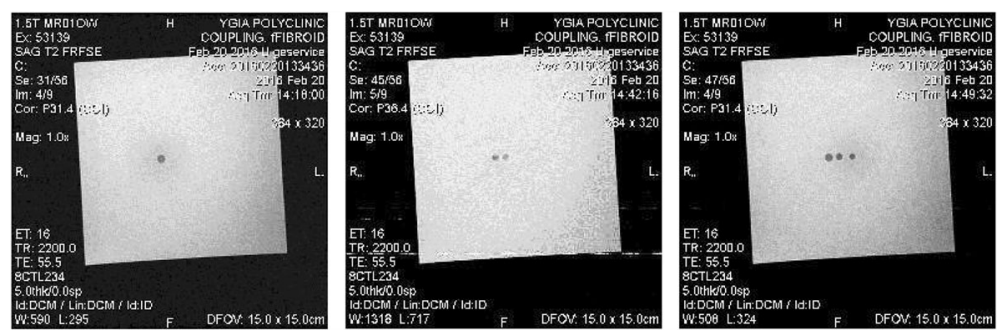

Fig. 8 MR images (using T2-W FSE) of three discrete thermal lesions created in the gel phantom by moving the $X$ stage of the robotic system. The acoustical power used was $30 \mathrm{~W}$ for $30 \mathrm{~s}$. The spatial step between lesions was $5 \mathrm{~mm}$

\section{Discussion}

Our study was inspired by several clinical trials that have shown that MRgFUS destroys fibroids [6, 7]. In these studies, the FUS transducer is placed in a water container which is integrated in the MRI table. The coupling with this technology is bottom to top, and the patient sits lying on the table in the prone position. The focal beam with the existing technologies is moved using phased-array technology which is very complicated and expensive. In this study, we presented an alternative technology of MRgFUS which is based on mechanical movement of a single-element spherically focused transducer. The proposed technology is simpler and cost-effective. The coupling to the target is top to bottom, and the patient may sit in supine position on the table. The major challenge of this technology is the coupling of the transducer to the target. This challenge has already been solved by several studies. The team of Theraclion "makes" a contact to the thyroid or to the breast [15] with a transducer placed on the top of the target reporting a more comfortable patient placement. This same concept was shown in another study performed by our group [16].

The major advantage of the proposed robot is that the patient can be placed on the MRI table in supine position. In the current systems, the patients are placed in the prone position. Since sometimes the treatment procedure can be long, lasting up to 3 hours per session $[17,18]$, the proposed system could provide better comfort for the patients. Additionally, the proposed system can access multiple anatomical locations.

Based on the American Society for Testing and Materials (ASTM) document F2503 described by Stoianovici et al. [19], the proposed system is classified as MRI-conditional because of the use of the FUS transducer, piezoelectric motors, and optical encoders. The piezoelectric motors, the transducer, and the optical encoders require the use of electricity and therefore, the system is MRI-conditional. Pneumatic systems [19] on the other hand are classified as MRI safe, because no electricity is used.

\section{Transducer}

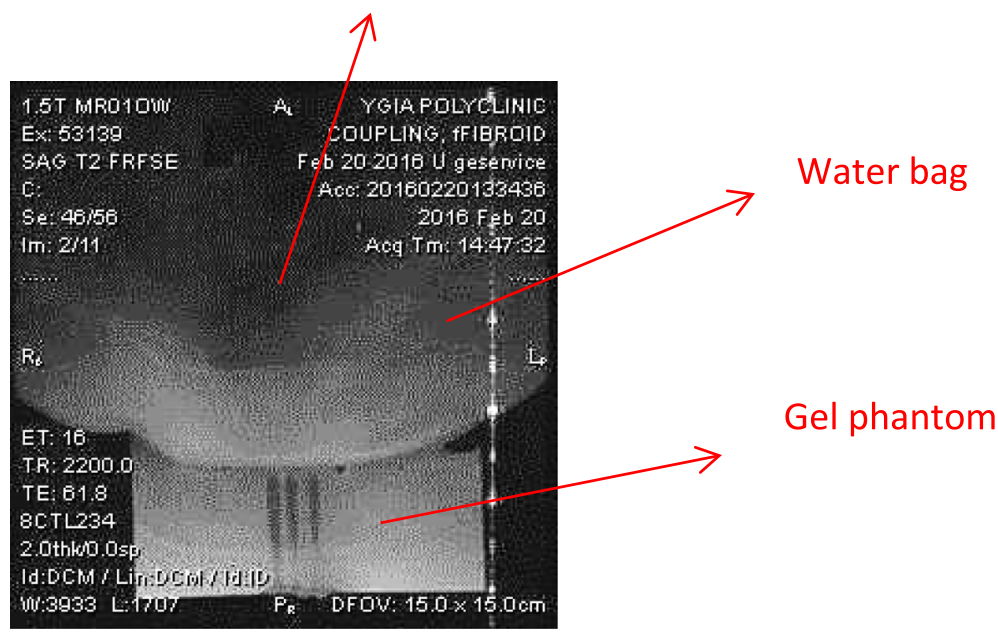

Fig. 9 MR image (using T2-W FSE) of the three discrete thermal lesions of Fig. 7 in axial plane demonstrating the penetration deep in the gel (plane perpendicular to the transducer face) 

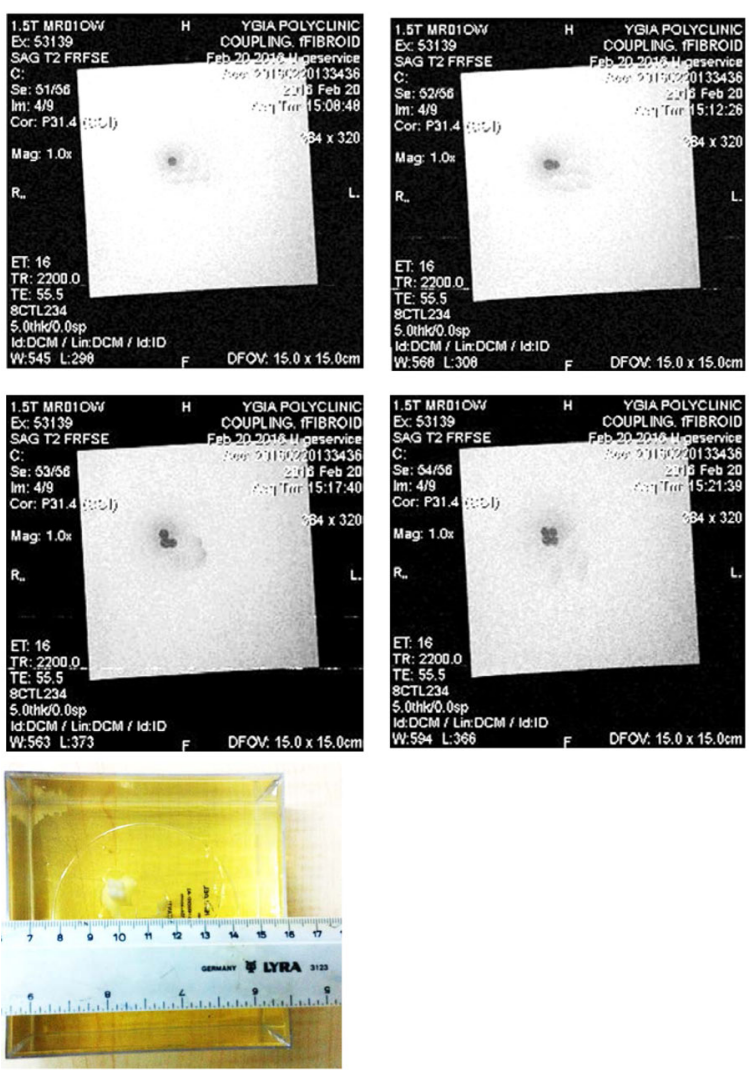

Fig. 10 MR images (using T2-W FSE) of four overlapping thermal lesions created in the gel phantom by moving the $X$ and $Y$ stages of the robotic system in a $2 \times 2$ square grid. The acoustical power used was $30 \mathrm{~W}$ for $30 \mathrm{~s}$. The spatial step between lesions was $3 \mathrm{~mm}$

\section{Conclusions}

The proposed technology is a continuation of other MRgFUS technologies designed by our group for various applications. Such system were developed for brain ablation using three Cartesian axes [12], prostate ablation using one linear and one angular axis [20, 21], and gynecological tumor ablation using one linear and one angular axis [22].

The FUS system produced lesions in gels successfully. The length and width of these lesions can be easily controlled by varying the intensity and time of exposure. These discrete and overlapped lesions were produced using the $X$ and $Y$ axes. The appearance of these lesions was demonstrated using MRI and proved that the linear stages moved with great accuracy. The degree of accuracy of the linear stages was also demonstrated in other articles of our group (for example, [12, 20, 21]). In future experiments, we plan to use thicker gel phantoms, in order to create lesions in a model which is closer to the size of anatomy involved for the case of fibroids.

In the future, some registration marks should be placed in the proposed system in order to transfer the position of the transducer or arm in the MRI images.
Additionally, an appropriate MRI coil should be selected. Currently, the lumbar spine was used (best option available to us). Better signal can be received if a dedicated coil is placed in proximity to the target. The proposed system which is modular can be easily modified to explore other applications with a top-to-bottom coupling arrangement (for example, breast or thyroid cancer). Finally, for better maneuverability, the proposed robot can be enhanced by the addition of additional motion stages to reach the performance reported by the FUSBOT robotic system [23].

\section{Abbreviations \\ ABS: Acrylonitrile butadiene styrene; ASTM: American Society for Testing and Materials; BW: Bandwidth; CE: Conformité Européenne; ETL: Echo train length; FSE: Fast spin echo; FUS: Focused ultrasound; GnRH: Gonadotrophin-releasing hormone; MRgFUS: Magnetic resonance-guided focused ultrasound system; MRI: Magnetic resonance imaging; NEX: Number of excitations; PRFS: Proton resonance frequency; ROI: Region of interest; SPGR: Spoiled gradient echo sequence; T2-W: T2-weighted; UAE: Uterine artery embolization}

\section{Acknowledgements}

Not applicable

\section{Funding}

This work was supported by the Project PROFUS E! 6620. PROFUS is implemented within the framework of the EUROSTARS Program and is co-funded by the European Community and the Research Promotion Foundation, under the EUROSTARS Cyprus Action of the EUREKA Cyprus Program (Project Code: EUREKA/EUSTAR/0311/01).

Availability of data and materials

The data will not be shared, because all the data is MRI data, which is of huge size and most of it is provided in the manuscript as images.

\section{Authors' contributions}

MY carried out the design of the positioning system. MY and CY carried out the design of the coupling for FUS using top-to-bottom propagation. GM designed and developed the MR thermometry software in MATLAB and did all the MRI work. CD performed the evaluation of the MRgFUS coupling for a focused ultrasound system using a top-to-bottom propagation system.

All authors read and approved the final manuscript.

\section{Competing interests}

The authors' declare that they have no competing interests.

\section{Consent for publication}

Not applicable.

\section{Ethics approval}

This article does not contain any studies with human participants or animals performed by any of the authors.

\section{Author details}

${ }^{1}$ Cyprus University of Technology, Limassol, Cyprus. ${ }^{2}$ City, University of London, London, UK. ${ }^{3}$ MEDSONIC LTD, Limassol, Cyprus.

Received: 19 May 2016 Accepted: 6 January 2017

Published online: 23 January 2017

\section{References}

1. Ravina JH, Ciraru-Vigneron N, Bouret JM, Herbreteau D, Houdart E, Aymard A, Merland JJ. Arterial embolisation to treat uterine myomata. Lancet. 1995;346(8976):671-2.

2. Weeks AD, Wilkinson N, Arora DS, Duffy SR, Wells M, Walker JJ. Menopausal changes in the myometrium: an investigation using a $\mathrm{GnRH}$ agonist model. Int J Gynecol Pathol. 1999;18(3):226-32.

3. Olive D, Rutherford T, Zreik T, Palter S. Cryomyolysis in the conservative treatment of uterine fibroids. J Am Assoc Gynecol Laparosc. 1996;3(4):S36. 
4. Zreik TG, Rutherford TJ, Palter SF, Troiano RN, Williams E, Brown JM, Olive DL. Cryomyolysis, a new procedure for the conservative treatment of uterine fibroids. J Am Assoc Gynecol Laparosc. 1998;5(1):33-8.

5. Zupi E, Piredda A, Marconi D, Townsend D, Exacoustos C, Arduini D, Szabolcs B. Directed laparoscopic cryomyolysis: a possible alternative to myomectomy and/or hysterectomy for symptomatic leiomyomas. Am J Obstet Gynecol. 2004;190(3):639-43.

6. Stewart EA, Gedroyc WMW, Tempany CMC, Quade BJ, Inbar Y, Ehrenstein T, Shushan A, Hindley JT, Goldin RD, David M, Sklair M, Rabinovici J. Focused ultrasound treatment of uterine fibroid tumors: safety and feasibility of a noninvasive thermoablative technique. Am J Obstet Gynecol. 2003;189(1):48-54.

7. Hesley GK, Felmlee JP, Gebhart JB, Dunagan KT, Gorny KR, Kesler JB, Brandt KR, Glantz JN, Gostout BS. Noninvasive treatment of uterine fibroids: early Mayo Clinic experience with magnetic resonance imaging-guided focused ultrasound. Mayo Clin Proc. 2006:81(7):936-42.

8. Mikami K, Murakami T, Okada A, Osuga K, Tomoda K, Nakamura H. Magnetic resonance imaging-guided focused ultrasound ablation of uterine fibroids: early clinical experience. Radiat Med. 2008;26(4):198-205.

9. Rabinovici J, David M, Fukunishi H, Morita Y, Gostout BS, Stewart EA. Pregnancy outcome after magnetic resonance-guided focused ultrasound surgery (MRgFUS) for conservative treatment of uterine fibroids. Fertil Steril. 2010:93(1):199-209.

10. Ikink ME, Voogt MJ, Verkooijen HM, Lohle PNM, Schweitzer KJ, Franx A, Mali WPTM, Bartels LW, van den Bosch MAAJ. Mid-term clinical efficacy of a volumetric magnetic resonance-guided high-intensity focused ultrasound technique for treatment of symptomatic uterine fibroids. Eur Radiol. 2013;23(11):3054-61.

11. Huisman M, van den Bosch MAAJ. MR-guided high-intensity focused ultrasound for noninvasive cancer treatment. Cancer Imaging. 2011;11:S161-6.

12. Mylonas N, Damianou C. MR compatible positioning device for guiding a focused ultrasound system for the treatment of brain diseases. Int J Med Robot. 2014;10(1):1-10.

13. Onda Corp. http://www.ondacorp.com/products_hifusol_phantoms.shtml. Accessed 1 Jan 2017.

14. Rieke V, Butts Pauly K. MR thermometry. J Magn Reson Imaging. 2008; 27(2):376-90.

15. Kovatcheva R, Guglielmina J-N, Abehsera M, Boulanger L, Laurent N, Poncelet E, Ultrasound-guided high-intensity focused ultrasound treatment of breast fibroadenoma—a multicenter experience. J Ther Ultrasound. 2015;3:1.

16. Damianou C, loannides K, Milonas N. Positioning device for MRI-guided high intensity focused ultrasound system. Int I Comput Assist Radiol Surg. 2008;2(6):335-45.

17. Abdullah B, Subramaniam R, Omar S, Wragg P, Ramli N, Wui A, Lee C, Yusof $Y$. Magnetic resonance-guided focused ultrasound surgery (MRgFUS) treatment for uterine fibroids. Biomed Imaging Interv J. 2010;6(2):e15.

18. Rueff LE, Raman SS. Clinical and technical aspects of MR-guided high intensity focused ultrasound for treatment of symptomatic uterine fibroids. Semin Intervent Radiol. 2013;30(4):347-53.

19. Stoianovici D, Kim C, Srimathveeravalli G, Sebrecht P, Petrisor D, Coleman J, Solomon SB, Hricak H. MRI-safe robot for endorectal prostate biopsy. IEEE ASME Trans Mechatron. 2013;19(4):1289-99.

20. Yiallouras C, Mylonas N, Damianou C. MRI-compatible positioning device for guiding a focused ultrasound system for transrectal treatment of prostate cancer. Int J Comput Assist Radiol Surg. 2014;9(4):745-53.

21. Yiallouras C, loannides K, Dadakova T, Pavlina M, Bock M, Damianou C. Three-axis MR-conditional robot for high-intensity focused ultrasound for treating prostate diseases transrectally. J Ther Ultrasound. 2015;3:2.

22. Epaminonda E, Drakos T, Kalogirou C, Theodoulou M, Yiallouras C, Damianou C. MRI guided focused ultrasound robotic system for the treatment of gynaecological tumors. Int J Med Robot. 2016;12(1):46-52.

23. Chauhan S, Amir H, Chen G, Hacker A, Michel MS, Koehrmann KU. Intra-operative feedback and dynamic compensation for image-guided robotic focal ultrasound surgery. Comput Aided Surg. 2008;13(6):353-68. doi:10.3109/10929080802586825.

\section{Submit your next manuscript to BioMed Central and we will help you at every step:}

- We accept pre-submission inquiries

- Our selector tool helps you to find the most relevant journal

- We provide round the clock customer support

- Convenient online submission

- Thorough peer review

- Inclusion in PubMed and all major indexing services

- Maximum visibility for your research

Submit your manuscript at www.biomedcentral.com/submit
Biomed Central 